Natural Hazards and Earth System Sciences (2003) 3: 777-780

(C) European Geosciences Union 2003

Natural Hazards and Earth System Sciences

Letter to the Editor

\title{
Analysis of natural neutron flux in a seismically active zone
}

\author{
V. F. Ostapenko ${ }^{1}$ and V. A. Krasnoperov ${ }^{2}$ \\ ${ }^{1}$ Institute of Seismology, Almaty, Kazakhstan \\ ${ }^{2}$ Suleyman Demirel University, Almaty, Kazakhstan
}

Received: 13 March 2003 - Revised: 06 July 2003 - Accepted: 14 September 2003

\begin{abstract}
In a seismically active zone in the near Almaty area (Kazakhstan) since 1996 observations of variations of a natural neutron flux have been conducted. Sometimes the neutron flux rises sharply within the one-hour interval in comparison with the background. It occurs on the eve of activation of seismic processes. Increase of the neutron flux level had taken place from $1 \mathrm{~h}$ to 10 days prior to earthquakes. It is also indicated a tendency of growth of the anomaly level in accordance with the growth of energetic class of the subsequent earthquake.

A character of connection between the neutron flux and earthquakes is still not clear. It is proposed that the neutron flux anomalies caused by variations of cosmic radiation intensity under action of fluxes of solar material, which is burst into interplanetary space (solar wind) during solar flares. Energy of the solar wind transferred to Earth puts into action a trigger mechanism of the process of initiation of earthquakes at those places where conditions have already been prepared for them.

The neutron flux anomalies can be used as substantial additional information for classical geophysical methods of short-term earthquake prediction.
\end{abstract}

\section{Results of experiments and conclusions}

Since 1996 we are carrying out continuous observations of the natural neutron flux in a drift near Almaty (Kazakhstan) at a height of $1600 \mathrm{~m}$ above sea level. The city of Almaty is situated at foothills of the Tien Shan, which is characterized by high seismic activity. Measurements are conducted with data accumulation during a one-hour period.

It was found that sometimes the neutron flux rises sharply within the one-hour interval in comparison with an ordinary one. Statistical distribution of dispersions of the neutron flux is close to normal. However, a quantity of dispersions from

Correspondence to: V. A. Krasnoperov

(vkras@academician.samal.kz) the average value, exceeding the latter more than by triple mean square error, are twice higher of theoretically expected value from Gaussian distribution. Such levels are considered as anomaly. Obviously there is a certain natural factor which causes such anomalies.

As is well known (see Gorshkov et al., 1966), the natural neutron flux consists of three components. First, neutrons are formed as a result of interaction of cosmic radiation with atomic nuclei of material of the atmosphere and the earth's crust. Secondly, free neutrons segregate during spontaneous fission of uranium nuclei. At last, neutrons can be generated during nuclear reactions when alpha-particles of natural radionuclides have been interacted with atomic nuclei of light elements of which the atmosphere and the earth's crust are consisted of.

Therefore, a changing of the neutron flux can be caused either by variations of intensity of cosmic radiation or by alteration of concentration of uranium and other radioelements emitting alpha-particles in the space adjacent to the place of position of a neutron detector. Comparison of measurement results showed that anomalous increase of the neutron flux could not be explained by an additional inflow of radon, which is the most mobile radioelement in the nature. The fact is that in the drift, which is tightly closed with doors, there is no ventilation of the air, so in case of additional inflow of radon neutron flux anomalies had to be observed during the time substantially exceeding the one hour interval as long as a half-value period amounts 3.8 days.

Contents of other radioelements in the ambient the neutron detector space is practically stable. Thus, a cause of changing of neutron flux most likely is the variations of cosmic radiation and in particular variations of $\mu$-mesons relating to the hard component thereof as since only these particles are able to penetrate the drift through tens meters of rock. Besides, the evaluation shows that in the conditions in the drift the neutron component formed under cosmic radiation comes to about $90 \%$ of the total registered underground neutron flux.

On analysis of measurement results, it was determined that there is a certain connection between the neutron flux and 


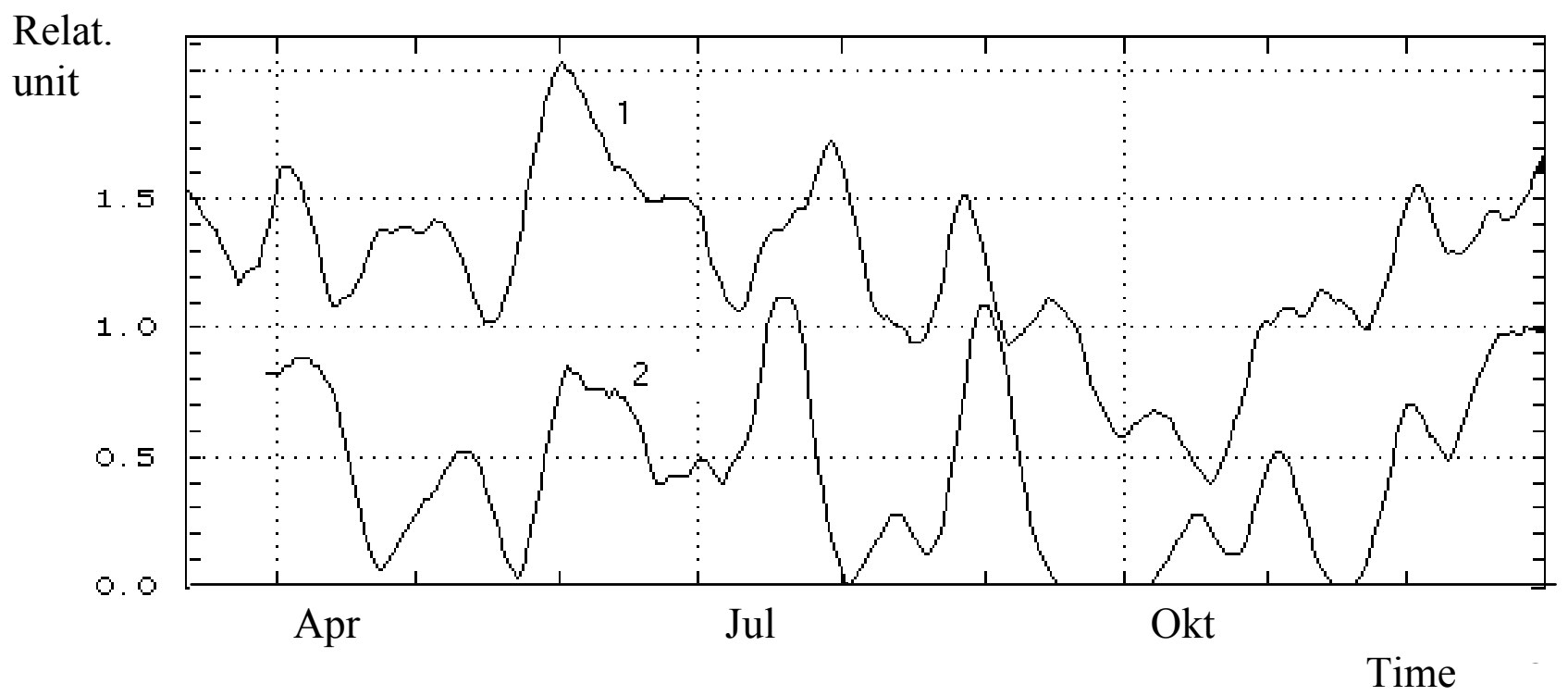

1996

Fig. 1. Smoothed curves of timing of moments of anomalies onset of the neutron flux (1) and earthquakes of energetic classes $\mathrm{K} \geq 10$ (2).

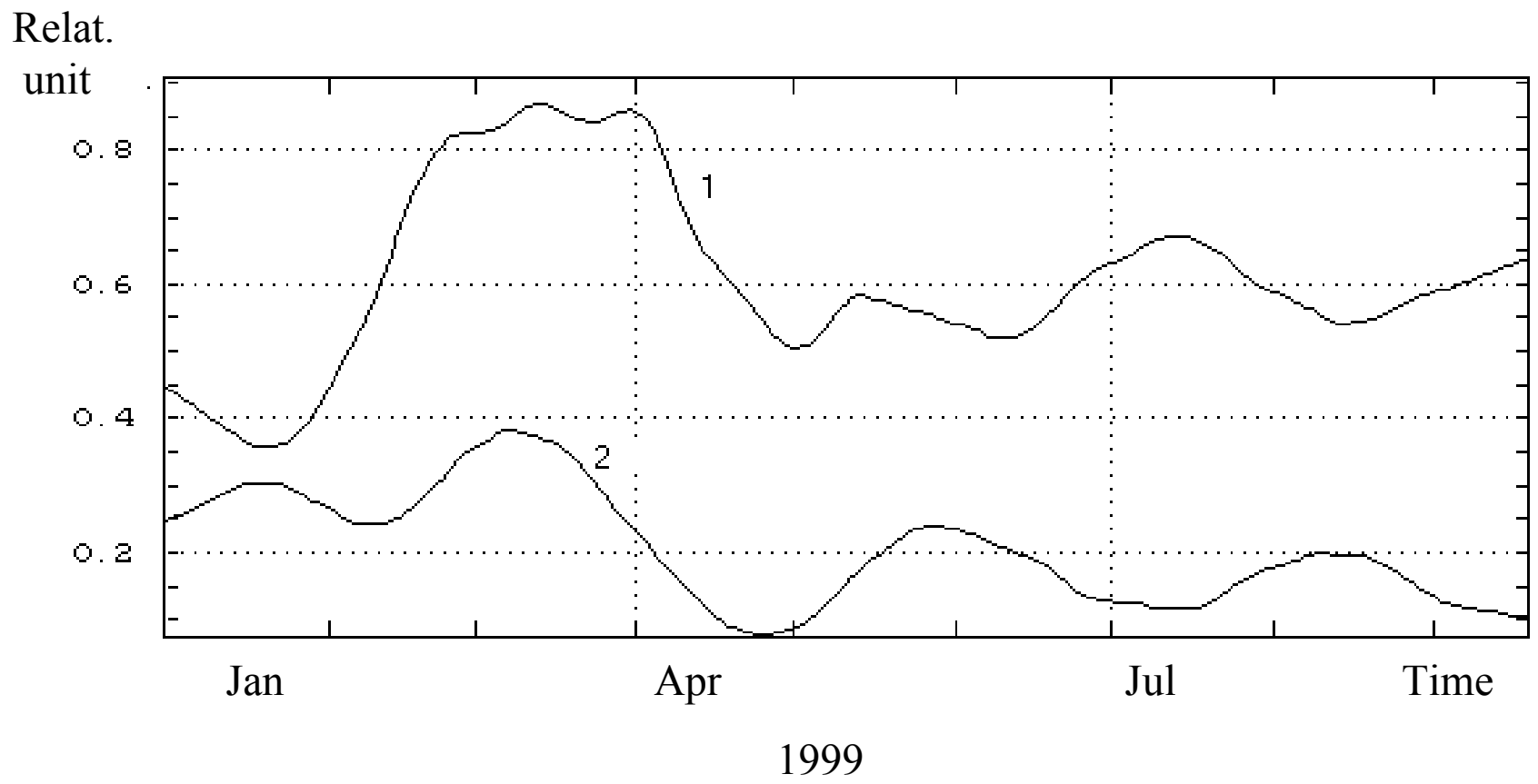

Fig. 2. The same as Fig. 1 but for the year 1999.

seismic events, which had been occurred within the range of about $450 \mathrm{~km}$ from the place of the neutron flux registration. For earthquakes occurred outside the $500 \mathrm{~km}$ zone anomalies were not observed. Periods of increase of frequency of anomalies usually coincides with periods of increase of seismic activity (see Figs. 1-2).

An earthquake level is measured by energetic class $\mathrm{K}$, which is equal to decimal logarithm of quantity of energy (in jouls) evolving during the earthquake.
Anomalous high values of the neutron flux registered within the one hour interval are accompanied as a rule by subsequent earthquakes of energetic classes $\mathrm{K} \geq 10$, epicenters of which are located within the range of $450 \mathrm{~km}$ from the place of the neutron flux registration. Figure 3 illustrates such an occurrence.

All the mentioned above is not in contradiction with the data published in literature. Thus, in the work of Armenian researchers (see Asatryan et al., 1991) it is stated that $30 \mathrm{~min}$ 


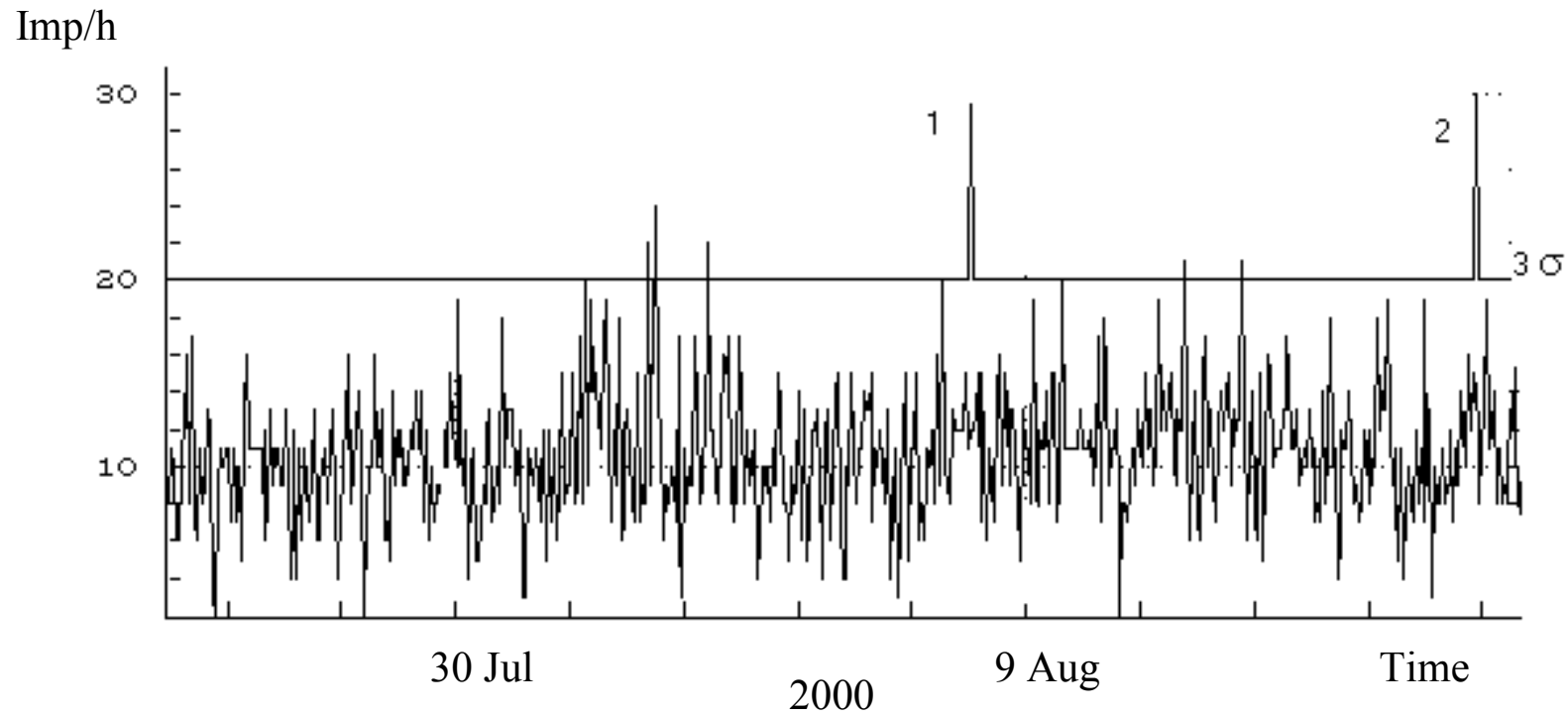

Fig. 3. A fragment of temporal series of the neutron flux. (1) and (2) are the moments of earthquakes of energetic classes 12.2 and 12.7, epicenters of which are located at the distance of $120 \mathrm{~km}$ and $335 \mathrm{~km}$ from the place of measurement respectively. Anomalies take place from $1 \mathrm{~h}$ to 10 days prior to earthquakes but mainly (up to $70 \%$ of cases) the time interval is not more than 4 days. It is also indicated a tendency of growth of the anomaly level in accordance with the growth of energetic class of the subsequent earthquake (Fig. 4).

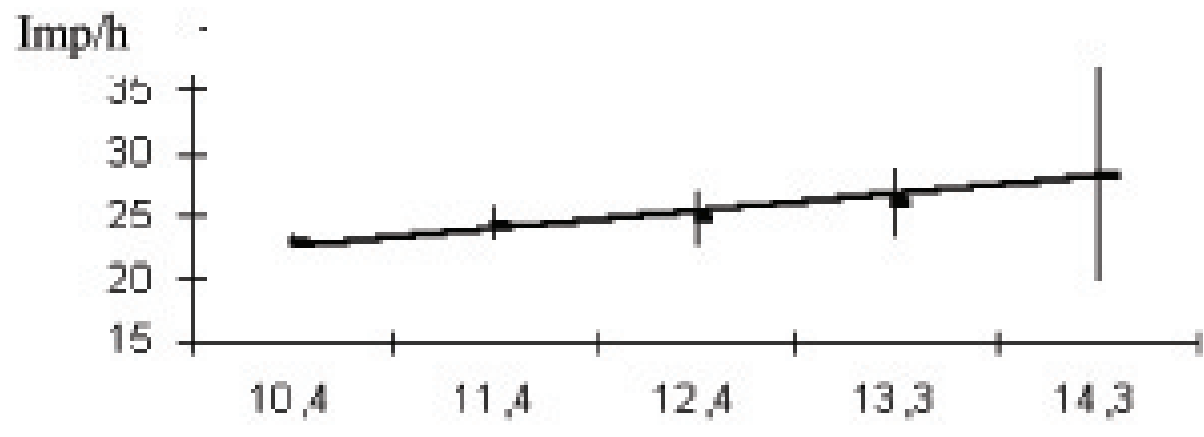

\section{Class}

Fig. 4. Amplitude dependence between anomalies of the neutron flux and the energetic class of earthquakes.

prior to the earthquake in Spitak, there has been registered the $100 \%$ increase of the hard component of cosmic radiation in stratosphere over Erevan. In another work (see Sobolev et al., 1998), it is distinguished a linear dependence between intensity of cosmic radiation registered by neutron monitors and the total energy-release caused by earthquakes all over the terrestrial globe. It is also indicated (see Karpov et al., 1997) that at Baksanskaya neutrino observatory there were registered short-term (not more than $15 \mathrm{~min}$ ) bursts of high energy $\mu$-mesons due to bursts of solar material into interplanetary space during solar flares. A character of connection between the neutron flux and earthquakes is still not clear. It is proposed that the neutron flux anomalies caused by variations of cosmic radiation intensity under action of fluxes of solar material, which is burst into interplanetary space (solar wind) during solar flares. Energy of the solar wind transferred to Earth puts into action a trigger mechanism of the process of initiation of earthquakes at those places where conditions have already been prepared for them.

During the period of observations within the range of $450 \mathrm{~km}$ from the place of location of the neutron detector is has been registered 44 earthquakes of energetic class varied from 12 to 12.9 and 18 earthquakes of energetic class $\mathrm{K} \geq 13$. All the earthquakes of the second group had been anticipated by neutron flux anomalies. Only 5 earthquakes in the first group took place without preliminary anomalies (see Table 1). As a consequence of all the stated above is a conclusion that neutron flux anomalies can be used as substantial additional information for classical geophysical methods of short-term earthquake prediction.

As a consequence of all the stated above is a conclusion that neutron flux anomalies can be used as substantial additional information for classical geophysical methods of short-term earthquake prediction. 
Table 1. Comparison of anomalies of the neutron flux with the subsequent earthquakes of energetic class $\mathrm{K} \geq 11$ for the period from 13 March 1996 to 18 November 2001.

\begin{tabular}{lccccccc}
\hline Years & 1996 & 1997 & 1998 & 1999 & 2000 & 2001 & Total \\
\hline Total quantity of anomalies & 329 & 310 & 272 & 206 & 82 & 26 & 1225 \\
\hline Quantity of false anomalies & 73 & 29 & 5 & 33 & 20 & 9 & 169 \\
\hline \% of false anomalies & 22 & 9 & 2 & 16 & 25 & 35 & 14 \\
\hline Total quantity of earthquakes of energetic class: & & & & & & & \\
$11-11.9$ & 12 & 46 & 47 & 18 & 17 & 9 & 149 \\
$12-12.9$ & 5 & 15 & 11 & 5 & 4 & 4 & 44 \\
$13-13.9$ & - & 6 & 6 & 1 & - & - & 13 \\
$\geq 14$ & 1 & 3 & 1 & - & - & - & 5 \\
\hline Total: & 18 & 70 & 65 & 21 & 13 & 211 & \\
\hline Missed earthquakes of energetic class: & & & & & & & 5 \\
$11-11.9$ & - & - & - & - & 1 & 5 & 6 \\
$12-12.9$ & - & - & 1 & - & 1 & 3 & 5 \\
$13-13.9$ & - & - & - & - & - & - & - \\
K $\geq 14$ & - & - & - & - & - & - & - \\
\hline
\end{tabular}

\section{References}

Asatryan, G. A., Asatryan, Gr. A., Babayan, V. H., Stozhkov, Yu. I., and Oganyan, G. Zh.: Proc. AS USSR, Ser. Phys., V. 55, No. 10, pp. 1979-1981, 1991.

Gorshkov, G. V., Zyabkin, V. A., Latkovskaya, N. M., and Tsvetkov, O. S.: Natural neutron background of the atmosphere and earth, Atomizdat, Moscow, pp. 178, 265, 333, 1966.

Karpov, S. N., Miroshnichenko, L. I., and Vashenyuk, E. V.: Proc. RAS, Ser. Phys. V. 61, No. 7, pp. 1466-1469, 1997.

Sobolev G. A., Shestopalov I. P., Kharin, E. P.: Geoeffective solar flares and seismic activity of the Earth, Physics of the Earth, No. 7, pp. 85-90, 1998. 